\title{
Hermitian scattering behavior for the non-Hermitian scattering center
}

\author{
L. Jin and Z. Song* \\ School of Physics, Nankai University, Tianjin 300071, China
}

\begin{abstract}
We study the scattering problem for the non-Hermitian scattering center, which consists of two Hermitian clusters with anti-Hermitian couplings between them. Counterintuitively, it is shown that it acts as a Hermitian scattering center, satisfying $|r|^{2}+|t|^{2}=1$, i.e., the Dirac probability current is conserved, when one of two clusters is embedded in the waveguides. This conclusion can be applied to an arbitrary parity-symmetric real Hermitian graph with additional $\mathcal{P} \mathcal{T}$-symmetric potentials, which is more feasible in experiment. Exactly solvable model is presented to illustrate the theory. Bethe ansatz solution indicates that the transmission spectrum of such a cluster displays peculiar feature arising from the non-Hermiticity of the scattering center.

PACS numbers: 11.30.Er, 03.65.Nk, 03.65.-w, 42.82.Et
\end{abstract}

\section{INTRODUCTION}

A non-Hermitian Hamiltonian is usually endowed with the physical meaning when it possesses entirely real quantum mechanical energy spectrum and the complex extension of the conventional quantum mechanics, a paritytime $(\mathcal{P} \mathcal{T})$ symmetric quantum theory, has been well developed [1 8 ] since the seminal discovery by Bender [1]. Such a theory gives the pseudo-Hermitian Hamiltonian a physical meaning via its corresponding Hermitian counterparts [9 11], which has an identical spectrum. The metric-operator theory outlined in Ref. [6] provides a mapping of such a pseudo-Hermitian Hamiltonian to an equivalent Hermitian Hamiltonian. Thus, most of the studies focused on the quasi-Hermitian system, or unbroken $\mathcal{P} \mathcal{T}$-symmetric region [12, 13]. However, the obtained equivalent Hermitian Hamiltonian is usually quite complicated [6, 14], involving long-range or nonlocal interactions, which is hardly realized in practice.

Experimentally, the $\mathcal{P} \mathcal{T}$ symmetry is of great relevance to the technological applications based on the fact that the imaginary potential could be realized by complex index in optics [15 18]. Furthermore, the $\mathcal{P} \mathcal{T}$ optical potentials can be realized through a judicious inclusion of index guiding and gain/loss regions. Such non-Hermitian systems are not isolated but usually embedded in the large Hermitian waveguides. Pure imaginary potential as a scattering center breaks the conservation of the flow of probability [19]. Thus, it is interesting to investigate what happens when the non-Hermitian system is with balanced gain and loss as a scattering center, and much effort devoted to such a topic is based on the framework of $\eta$-metric 20 23.

In this paper, we study the scattering problem for the non-Hermitian scattering center based on the configurations involving two arbitrary Hermitian networks coupled with anti-Hermitian interaction. It is shown that for any scattering state of such a non-Hermitian system, the Dirac probability current is always conserved at any

* songtc@nankai.edu.cn degree of the non-Hermiticity. We apply such a rigorous result to the system with $\mathcal{P} \mathcal{T}$-symmetric potentials, which is more feasible in experiment.

This paper is organized as follows. Section $\llbracket$ presents the exact analytical solution of the scattering problem for the concerned non-Hermitian scattering center. Section III is the application of the rigorous result to the system with $\mathcal{P} \mathcal{T}$-symmetric potentials. Section IV consists of an exactly solvable example to illustrate our main idea. Section $\square$ is the summary and discussion.

\section{MODEL AND FORMALISM}

In general, a non-Hermitian Hamiltonian $H$ is related by a similarity transformation to an equivalent Hermitian Hamiltonian $h$. Such a connection is valid within the so called unbroken symmetric region. However, when a non-Hermitian system interacts with other Hermitian system, such a region loses its physical meaning: On the one hand, the unbroken symmetric region is shifted in the whole non-Hermitian system. On the other hand, it may act as a Hermitian system in the scattering problem without the restriction on the degree of the non-Hermiticity. In this section, we will investigate the latter situation.

The Hamiltonian of the concerned scattering tightbinding network has the form

$$
H=H_{L}+H_{R}+H_{C},
$$

where

$$
\begin{aligned}
& H_{L}=-\kappa \sum_{j=-1}^{-\infty}|j\rangle_{L}\left\langle j-1\left|-g_{L}\right| L\right\rangle_{L}\langle-1|+\text { H.c. } \\
& H_{R}=-\kappa \sum_{j=1}^{+\infty}|j\rangle_{R}\left\langle j+1\left|-g_{R}\right| R\right\rangle_{R}\langle 1|+\text { H.c. }
\end{aligned}
$$

represent the left $\left(H_{L}\right)$ and right $\left(H_{R}\right)$ waveguides with real $\kappa$ and

$$
H_{C}=H_{A}+H_{B}+H_{A B}+H_{B A},
$$




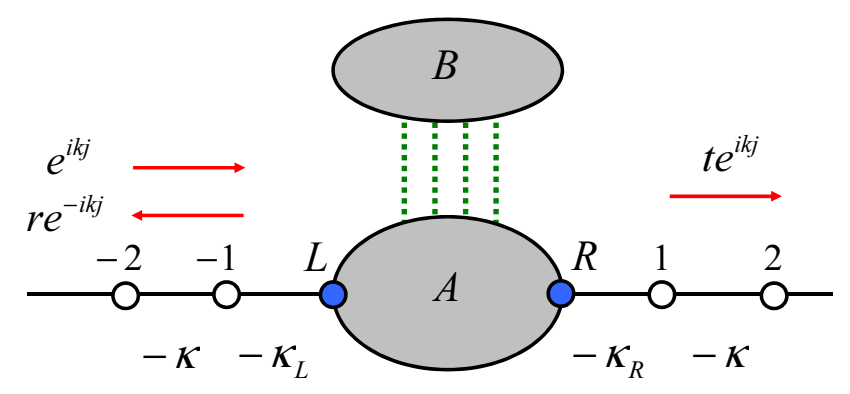

FIG. 1. ((Color online) Schematic illustration of the configuration of the concerned network. It consists of two arbitrary graphs of the Hermitian tight-binding networks $A$ and $B$ (shadow) with one of them connecting to two semi-infinite chains as the waveguides at the joint sites $L$ and $R$. The nonHermiticity of the whole scattering center $A+B$ arises from the anti-Hermitian interaction (dished lines) between them. It is shown that the non-Hermitian scattering center acts as a Hermitian one, preserving the Dirac probability current.

describes a non-Hermitian network as a scattering center. Here $|L\rangle$ and $|R\rangle$ denote the sites state on the joint sites on the network $A$, which are simply taken as $|L\rangle=|1\rangle_{A}$ and $|R\rangle=\left|N_{A}\right\rangle_{A}$ without losing the generality. The subgraphs

$$
\begin{aligned}
& H_{A}=\sum_{i, j=1}^{N_{A}}\left(H_{A}\right)_{i j}|i\rangle_{A}\langle j|, \\
& H_{B}=\sum_{i, j=1}^{N_{B}}\left(H_{B}\right)_{i j}|i\rangle_{B}\langle j|,
\end{aligned}
$$

are arbitrary Hermitian networks, i.e., $H_{A}^{\dagger}=H_{A}$, and $H_{B}^{\dagger}=H_{B}$, while the coupling between them is antiHermitian, i.e., $H_{A B}^{\dagger}=-H_{B A}$.

$$
H_{A B}=\sum_{i=1}^{N_{A}} \sum_{j=1}^{N_{B}}\left(H_{A B}\right)_{i j}|i\rangle_{A B}\langle j|
$$

then the scattering center with respect to the basis $\left\{|i\rangle_{A},|i\rangle_{B}\right\}$ is in the form of

$$
H_{C}=\left(\begin{array}{cc}
H_{A} & H_{A B} \\
-H_{A B}^{\dagger} & H_{B}
\end{array}\right)
$$

The non-Hermiticity of $H_{C}$ arises from this antiHermitian term. The non-Hermitian Hamiltonian $H_{C}$ may have fully real spectrum or not. In the following, we will show that it always acts as a Hermitian scattering center no matter the reality of the spectrum.

For an incident plane wave with momentum $k$ incoming from the left waveguide $L$ with energy $E=-2 \kappa \cos k$, the scattering wave function $\left|\psi_{k}\right\rangle$ can be obtained by the Bethe ansatz method. The wave function has the form

$$
\left|\psi_{k}\right\rangle=\sum_{j=-1}^{-\infty} f_{j}|j\rangle_{L}+\sum_{j=1}^{N_{A}} \alpha_{j}|j\rangle_{A}+\sum_{j=1}^{N_{B}} \beta_{j}|j\rangle_{B}+\sum_{j=1}^{+\infty} f_{j}|j\rangle_{R}
$$

where the scattering wavefunction $f_{j}$ is in form of

$$
f_{j}=\left\{\begin{array}{c}
e^{i k j}+r e^{-i k j},(j \leqslant-1) \\
t e^{i k j},(j \geqslant 1)
\end{array} .\right.
$$

Here $r, t$ are the reflection and transmission coefficients of the incident wave, which is what we concern only in this paper. Substituting the wavefunction $\left|\psi_{k}\right\rangle$ into the Schrödinger equation

$$
H\left|\psi_{k}\right\rangle=E\left|\psi_{k}\right\rangle
$$

the explicit form of the Schrödinger equations in the truncated Hilbert space spanned by the basis $\left\{\left|j, j \in\left[1, N_{A}\right]\right\rangle_{A},\left|j, j \in\left[1, N_{B}\right]\right\rangle_{B}\right\}$ can be expressed in the following matrix equation form

$$
\Delta\left(\begin{array}{c}
\alpha_{1} \\
\vdots \\
\alpha_{j} \\
\vdots \\
\alpha_{N_{A}} \\
\beta_{1} \\
\vdots \\
\beta_{j} \\
\vdots \\
\beta_{N_{B}}
\end{array}\right)=\left(\begin{array}{c}
g_{L} f_{-1} \\
0 \\
\vdots \\
0 \\
g_{R} f_{1} \\
0 \\
\vdots \\
0 \\
\vdots \\
0
\end{array}\right),
$$

where $\Delta$ is an $\left(N_{A}+N_{B}\right) \times\left(N_{A}+N_{B}\right)$ matrix defined by

$$
\Delta=\left(\begin{array}{cc}
H_{A}-E & H_{A B} \\
-H_{A B}^{\dagger} & H_{B}-E
\end{array}\right)
$$

From the reduced Schrödinger equation of Eq. (12), we obtain

$$
\begin{aligned}
\alpha_{1} & =\left(\Delta^{-1}\right)_{11} g_{L} f_{-1}+\left(\Delta^{-1}\right)_{1 N_{A}} g_{R} f_{1}, \\
\alpha_{N_{A}} & =\left(\Delta^{-1}\right)_{N_{A} 1} g_{L} f_{-1}+\left(\Delta^{-1}\right)_{N_{A} N_{A}} g_{R} f_{1},
\end{aligned}
$$

Here $\Delta^{-1}$ is the inverse of matrix $\Delta$, with the element being expressed as

$$
\left(\Delta^{-1}\right)_{i j}=\frac{C_{j i}}{\operatorname{det}(\Delta)}=\frac{(-1)^{i+j} \operatorname{det}\left(M_{j i}\right)}{\operatorname{det}(\Delta)},
$$

in term of the matrix of cofactors $C_{i j}$. Here $M_{i j}$ is the matrix obtained by deleting the $i$ th row and $j$ th column 
from the matrix $\Delta$. On the other hand, the Schrödinger equations for the sites of the waveguides connecting to the joints of the scattering center are

$$
\begin{aligned}
& -\kappa f_{-2}-g_{L}^{*} \alpha_{1}=E f_{-1}, \\
& -\kappa f_{2}-g_{R}^{*} \alpha_{N_{A}}=E f_{1},
\end{aligned}
$$

which lead to

$$
\alpha_{1}=\frac{\kappa}{g_{L}^{*}}(1+r), \alpha_{N_{A}}=\frac{\kappa}{g_{R}^{*}} t .
$$

Then associating with Eqs. (14), we have

$$
\begin{aligned}
r & =\left(-b \widetilde{b}+a c-a e^{-i k}-c e^{i k}+1\right) / \eta, \\
t & =i 2 \widetilde{b} \sin k / \eta,
\end{aligned}
$$

where

$$
\begin{aligned}
\eta & =(b \widetilde{b}-a c) e^{i 2 k}+(a+c) e^{i k}-1, \\
a & =\left(\Delta^{-1}\right)_{11}\left|g_{L}\right|^{2} / \kappa, c=\left(\Delta^{-1}\right)_{N_{A} N_{A}}\left|g_{R}\right|^{2} / \kappa, \\
b & =\left(\Delta^{-1}\right)_{1 N_{A}} g_{L}^{*} g_{R} / \kappa, \widetilde{b}=\left(\Delta^{-1}\right)_{N_{A} 1} g_{L} g_{R}^{*} / \kappa .
\end{aligned}
$$

One can determine the unknown coefficients $a, b, \widetilde{b}, c$ and $\eta$ through the matrix $\Delta$ by requiring that invertible matrix $\left(H_{A}-E\right)$ or $\left(H_{B}-E\right)$ exists.

In the Appendix, we will show that

$$
\left(\Delta^{-1}\right)_{i j}=\left(\Delta^{-1}\right)_{j i}^{*}
$$

for $i, j \in\left[1, N_{A}\right]$, or more explicitly for special cases

$$
\begin{aligned}
\left(\Delta^{-1}\right)_{11} & =\left(\Delta^{-1}\right)_{11}^{*}, \\
\left(\Delta^{-1}\right)_{N_{A} N_{A}} & =\left(\Delta^{-1}\right)_{N_{A} N_{A}}^{*}, \\
\left(\Delta^{-1}\right)_{1 N_{A}} & =\left(\Delta^{-1}\right)_{N_{A} 1}^{*},
\end{aligned}
$$

which indicate that both $a$ and $c$ are real, and $\widetilde{b}=b^{*}$. It is somewhat surprising that we get the conclusion from Eqs. (18), (19), (20) and (22) that

$$
|r|^{2}+|t|^{2}=1,
$$

which is common phenomenon in a Hermitian system but surprising in a non-Hermitian system.

\section{III. $\quad \mathcal{P}$ T-SYMMETRIC POTENTIALS}

The accessible setup of non-Hermitian system in the lab is the $\mathcal{P} \mathcal{T}$-symmetric potentials, which can be realized through a judicious inclusion of index guiding and gain/loss regions. In the following, we will apply the obtained result to the system with the $\mathcal{P} \mathcal{T}$-symmetric potentials, in which the $\mathcal{P} \mathcal{T}$-symmetrical axis is along the waveguides.
The geometry of the scattering center contains $N_{1}+$ $2 N_{2}$ sites and possesses the following symmetry,

$$
\begin{aligned}
& \mathcal{P}:|j\rangle_{c} \longrightarrow|j\rangle_{c}, \quad\left(j \in\left[1, N_{1}\right]\right) \\
& \mathcal{P}:|j\rangle_{c} \longrightarrow|j\rangle_{c}=\left|N_{2}+j\right\rangle_{c}, \quad\left(j-N_{1} \in\left[1, N_{2}\right]\right)
\end{aligned}
$$

with the joint points $L, R \in\left[1, N_{1}\right]$, where ${\overline{|j\rangle_{c}}}_{c}$ is the mirror symmetric counterpart of state $|j\rangle_{c}$. We define the Hamiltonian of the center has the form

$$
\begin{aligned}
& H_{\mathcal{P} \mathcal{T}}=\sum_{i, j=1,(i<j)}^{N_{1}+2 N_{2}} \kappa_{i j}|i\rangle_{c}\langle j|+\text { H.c. } \\
& +\sum_{j=1}^{N_{1}} U_{j}|j\rangle_{c}\langle j|+\sum_{j=N_{1}+1}^{N_{1}+N_{2}}\left(V_{j}|j\rangle_{c}\langle j|+V_{j}^{*} \overline{|j\rangle_{c}} \overline{\langle j|}\right),
\end{aligned}
$$

where $\kappa_{i j}$ and $U_{j}$ are real. In the Hilbert space spanned by basis $\left\{|j\rangle_{c}\right\} \quad\left(j \in\left[1, N_{1}+2 N_{2}\right]\right)$, the matrix of the Hamiltonian $H_{\mathcal{P} \mathcal{T}}$ has the form

$$
H_{\mathcal{P} \mathcal{T}}=\left(\begin{array}{ccc}
H_{\gamma} & H_{\gamma \alpha} & H_{\gamma \alpha} \\
H_{\gamma \alpha}^{\dagger} & H_{\alpha}+H_{\delta} & H_{\alpha \beta} \\
H_{\gamma \alpha}^{\dagger} & H_{\alpha \beta} & H_{\alpha}-H_{\delta}
\end{array}\right),
$$

where

$$
\left(H_{\delta}\right)_{i j}=\delta_{i j}\left(V_{j}-V_{j}^{*}\right) / 2=i \operatorname{Im}\left(V_{j}\right) \delta_{i j}
$$

and $H_{\gamma}\left(H_{\alpha}\right)$ is an $N_{1}\left(N_{2}\right)$ dimension square matrix. The matrices $H_{\gamma}, H_{\alpha}$, and $H_{\alpha \beta}$ are all real Hermitian while $H_{\gamma \alpha}$ is real. We can see that Hamiltonian $H_{\mathcal{P} \mathcal{T}}$ describes an arbitrary real Hermitian graph with paritysymmetry as defined in Eq. (24) combining with the onsite $\mathcal{P} \mathcal{T}$-symmetric potentials $H_{\delta}$. Thus $H_{\mathcal{P} \mathcal{T}}$ satisfies $\left[\mathcal{P} \mathcal{T}, H_{\mathcal{P} \mathcal{T}}\right]=0$.

Introducing the linear transformation

$$
\begin{aligned}
|j\rangle_{A} & =\left\{\begin{array}{cc}
|j\rangle_{c}, & \left(j \in\left[1, N_{1}\right]\right) \\
\left(|j\rangle_{c}+{\overline{|j\rangle_{c}}}_{c}\right) / \sqrt{2}, & \left(j-N_{1} \in\left[1, N_{2}\right]\right)
\end{array}\right. \\
|j\rangle_{B} & =\left(|j\rangle_{c}-\overline{|j\rangle_{c}}\right) / \sqrt{2}, \quad\left(j-N_{1} \in\left[1, N_{2}\right]\right)
\end{aligned}
$$

one can rewrite the matrix of Eq. (26) in the basis $\left\{\left|j, j \in\left[1, N_{1}+N_{2}\right]\right\rangle_{A},\left|j, j \in\left[N_{1}+1, N_{1}+N_{2}\right]\right\rangle_{B}\right\}$ as the form

$$
H_{\mathcal{P} \mathcal{T}}=\left(\begin{array}{ccc}
H_{\gamma} & \sqrt{2} H_{\gamma \alpha} & 0 \\
\sqrt{2} H_{\gamma \alpha}^{\dagger} & H_{\alpha}+H_{\alpha \beta} & H_{\delta} \\
0 & H_{\delta} & H_{\alpha}-H_{\alpha \beta}
\end{array}\right)
$$

Obviously, it is the special case of Eq. (8), where

$$
\begin{aligned}
H_{A} & =\left(\begin{array}{cc}
H_{\gamma} & \sqrt{2} H_{\gamma \alpha} \\
\sqrt{2} H_{\gamma \alpha}^{\dagger} & H_{\alpha}+H_{\alpha \beta}
\end{array}\right), \\
H_{B} & =H_{\alpha}-H_{\alpha \beta}, \\
H_{A B} & =\left(\begin{array}{c}
0 \\
H_{\delta}
\end{array}\right), H_{B A}=\left(\begin{array}{ll}
0 & H_{\delta}
\end{array}\right),
\end{aligned}
$$



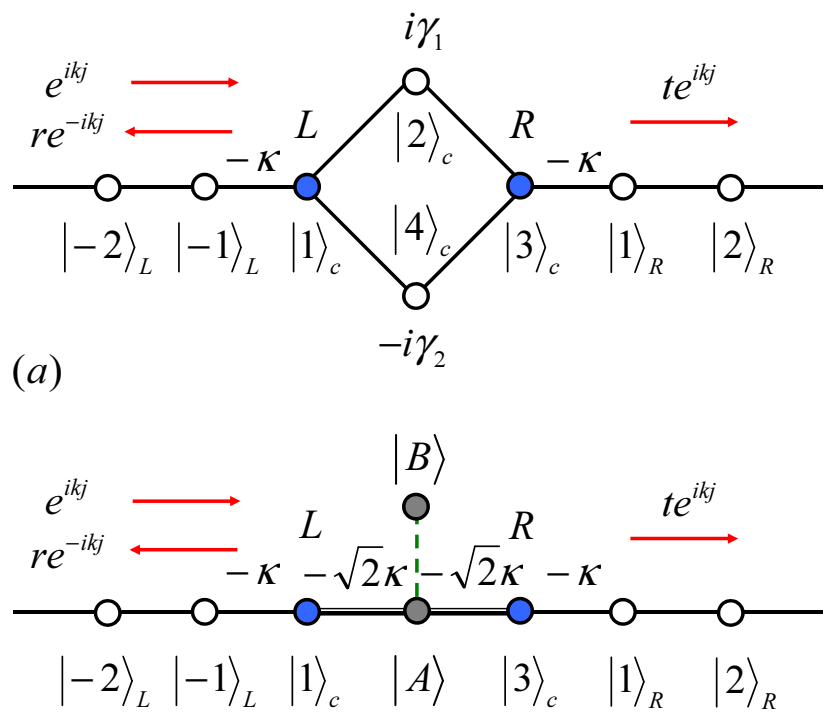

(b)

FIG. 2. (Color online) Schematic illustration for the exemplified system. (a) 4-site non-Hermitian scattering center configuration, which consists of two on-site imaginary potentials $i \gamma_{1}$ and $-i \gamma_{2}$ connecting to two semi-infinite chains as the waveguides at the joint sites $|1\rangle_{c}$ and $|3\rangle_{c}$, with the hopping strength $-\kappa$. (b) The equivalent Hamiltonian of $H_{C}$ [Eq. (48)], which is obtained under the linear transformation of Eq. (47). The hopping strengths between the site $|A\rangle$ and $|1\rangle_{c},|3\rangle_{c}$ are both $-\sqrt{2} \kappa$. The dashed (green) line represents the effective hopping between sites $|A\rangle$ and $|B\rangle$ which is pure imaginary $i\left(\gamma_{1}+\gamma_{2}\right) / 2$, with both potentials on $|A\rangle$ and $|B\rangle$ being $i\left(\gamma_{1}-\gamma_{2}\right) / 2$. In the case of $\gamma_{1}=\gamma_{2}$, it is shown that the non-Hermitian scattering center acts as a Hermitian one, preserving the Dirac probability current.

or equivalently in the explicit form as

$$
\begin{aligned}
\left(H_{A}\right)_{m n} & ={ }_{A}\left\langle m\left|H_{\mathcal{P} \mathcal{T}}\right| n\right\rangle_{A}=\left(H_{A}\right)_{n m}^{*}, \\
\left(H_{B}\right)_{m n} & ={ }_{B}\left\langle m\left|H_{\mathcal{P} \mathcal{T}}\right| n\right\rangle_{B}=\left(H_{B}\right)_{n m}^{*}, \\
\left(H_{A B}\right)_{m n} & ={ }_{A}\left\langle m\left|H_{\mathcal{P} \mathcal{T}}\right| n\right\rangle_{B}=i \operatorname{Im}\left(V_{m}\right) \delta_{m-N_{1}, n}, \\
\left(H_{B A}\right)_{m n} & ={ }_{B}\left\langle m\left|H_{\mathcal{P} \mathcal{T}}\right| n\right\rangle_{A}=i \operatorname{Im}\left(V_{n}\right) \delta_{m, n-N_{1}} .
\end{aligned}
$$

Therefore, the cluster $H_{\mathcal{P} \mathcal{T}}$ acts as a Hermitian scattering center. This result is independent of the magnitudes of the hopping integrals and the potentials, also the reality of the spectrum of $H_{\mathcal{P} \mathcal{T}}$.

\section{ILLUSTRATION}

We consider a simple 4-site non-Hermitian scattering center which is illustrated schematically in Fig. 2(a). The Hamiltonian of the whole system of Eq. (1) can be written as

$$
\begin{aligned}
& H_{L}=-\kappa \sum_{j=-1}^{-\infty}|j\rangle_{L}\langle j-1|-\kappa|-1\rangle_{L c}\langle 1|+\text { H.c., } \\
& H_{R}=-\kappa \sum_{j=1}^{+\infty}|j\rangle_{R}\langle j+1|-\kappa| 1\rangle_{R c}\langle 3|+\text { H.c., }
\end{aligned}
$$

where the joints of the scattering center are $L=1, R=3$ and

$$
\begin{aligned}
H_{C}= & -\kappa\left(|1\rangle_{c}\langle 2|+| 2\rangle_{c}\langle 3|+| 3\rangle_{c}\langle 4|+| 4\rangle_{c}\langle 1|+\text { H.c. }\right) \\
& +i \gamma_{1}|2\rangle_{c}\left\langle 2\left|-i \gamma_{2}\right| 4\right\rangle_{c}\langle 4| .
\end{aligned}
$$

Note that here we consider a non- $\mathcal{P} \mathcal{T}$-symmetric model without losing the generality. The Bethe ansatz wavefunction has the form

$$
\left|\phi_{k}\right\rangle=\sum_{j=-1}^{-\infty} f_{j}|j\rangle_{L}+\sum_{j=1}^{4} h_{j}|j\rangle_{c}+\sum_{j=1}^{+\infty} f_{j}|j\rangle_{R},
$$

where $f_{j}$ is in form of Eq. (10). Taking $\kappa=1$, the explicit form of Schrödinger equations are

$$
\begin{aligned}
-f_{-1}-h_{2}-h_{4} & =E h_{1}, \\
-f_{1}-h_{2}-h_{4} & =E h_{3}, \\
-h_{1}-h_{3} & =\left(E-i \gamma_{1}\right) h_{2}, \\
-h_{1}-h_{3} & =\left(E+i \gamma_{2}\right) h_{4}, \\
E & =-2 \cos k .
\end{aligned}
$$

the continuity of the wavefunctions demands

$$
h_{1}=1+r, h_{3}=t,
$$

The corresponding transmission and reflection coefficients have the form

$$
\begin{aligned}
& r=\frac{\zeta \cos k-1}{e^{-i k}-\zeta} e^{i k} \\
& t=-\frac{i \zeta \sin k}{e^{-i k}-\zeta} e^{i k}
\end{aligned}
$$

where

$$
\zeta=\frac{1}{\cos k+i \gamma_{1} / 2}+\frac{1}{\cos k-i \gamma_{2} / 2}
$$

Straightforward algebra shows that

$$
|r|^{2}+|t|^{2}=1-\frac{2 \operatorname{Im}(\zeta) \sin k}{1+|\zeta|^{2}-2 \operatorname{Re}(\zeta) \cos k+2 \operatorname{Im}(\zeta) \sin k},
$$

which indicates the current is conserved when $\zeta$ is real, i.e., $\gamma_{1}=\gamma_{2}$. 
Alternatively, taking the linear transformation

$$
\begin{aligned}
& |A\rangle=\left(|2\rangle_{c}+|4\rangle_{c}\right) / \sqrt{2} \\
& |B\rangle=\left(|2\rangle_{c}-|4\rangle_{c}\right) / \sqrt{2}
\end{aligned}
$$

the Hamiltonian $H_{C}$ can be rewritten as

$$
\begin{aligned}
H_{C}= & -\sqrt{2} \kappa\left(|A\rangle_{c}\langle 1|+| A\rangle_{c}\langle 3|+\text { H.c. }\right) \\
& +i \frac{\left(\gamma_{1}-\gamma_{2}\right)}{2}(|A\rangle\langle A|+| B\rangle\langle B|) \\
& +i \frac{\left(\gamma_{1}+\gamma_{2}\right)}{2}(|A\rangle\langle B|+| B\rangle\langle A|),
\end{aligned}
$$

as illustrated schematically in Fig. 2(b). It depicts a scattering configuration with single side coupling site which has been systematically studied in the Hermitian regime [24]. Obviously, when $\gamma_{1}=\gamma_{2}=\gamma$, it is a simple example of a real Hermitian graph with parity-symmetry combining with the on-site $\mathcal{P} \mathcal{T}$-symmetric potentials and admits the current preserving.

Accordingly, the transmission probability (coefficient) has the form

$$
T(k)=\frac{\sin ^{2}(2 k)}{\sin ^{2}(2 k)+\left(\cos ^{2}(k)-\gamma^{2} / 4\right)^{2}},
$$

which has peculiar feature in contrast to that of Hermitian scattering center. As a comparison, we write the transmission probability for the real side coupling by substituting $\gamma$ with $i \gamma$, i.e.,

$$
T^{\prime}(k)=\frac{\sin ^{2}(2 k)}{\sin ^{2}(2 k)+\left(\cos ^{2}(k)+\gamma^{2} / 4\right)^{2}} .
$$

It can be observed that, (i) both of them have the common total reflection points, $T(\pi / 2)=T^{\prime}(\pi / 2)=0$; (ii) $T(k)=1$ at the resonance condition $\gamma=2|\cos k|$, while $T^{\prime}(k)$ is always less than 1 within the whole range of $\gamma$.

\section{SUMMARY AND DISCUSSION}

We have proposed an anti-Hermitian coupled two Hermitian graphs as the scattering center, which has been shown to act as a Hermitian graph, preserving the traditional probability. This conclusion can be applied to the non-Hermitian scattering center which consists of pairs of $\mathcal{P} \mathcal{T}$-symmetric on-site potentials. This fact indicates the balanced gain and loss can result in the Hermiticity of the scattering center. Our results can give a good prediction for the transmission and reflection coefficients of linear waves scattered at the $\mathcal{P} \mathcal{T}$-symmetric defects in the experiment. The recent observation of breaking of $\mathcal{P} \mathcal{T}$ symmetry in coupled optical waveguides [25-27] may pave the way to demonstrate the result presented in this paper.

Finally, we would like to point that our conclusion can also apply to other type of non-Hermitian scattering center. For instance, we can select $H_{\gamma}, H_{\alpha}, H_{\gamma \alpha}$ and $H_{\alpha \beta}$ being all Hermitian instead of real Hermitian in Eq. (26), and we note that $H_{\mathcal{P} \mathcal{T}}$ is no longer $\mathcal{P} \mathcal{T}$-symmetric if the hoppings are not all real, with $H_{\gamma}, H_{\alpha}, H_{\gamma \alpha}$ and $H_{\alpha \beta}$ being Hermitian, we could also select $H_{\mathcal{P} \mathcal{T}}$ as

$$
H_{\mathcal{P} \mathcal{T}}=\left(\begin{array}{ccc}
H_{\gamma} & H_{\gamma \alpha} & H_{\gamma \alpha} \\
H_{\gamma \alpha}^{\dagger} & H_{\alpha}+H_{\delta} & H_{\alpha \beta} \\
H_{\gamma \alpha}^{\dagger} & H_{\alpha \beta}^{*} & H_{\alpha}-H_{\delta}
\end{array}\right)
$$

which is also in form of Eq. (8) after the transformation of Eq. (28) and exhibits the Hermitian behavior.

\section{APPENDIX}

In this Appendix, we will prove the relation of Eq. (21). For an incident plane wave with real energy $E$, we obtain from Eq. (13) and $H_{A}=H_{A}^{\dagger}, H_{B}=H_{B}^{\dagger}$ that

$$
\Delta^{\dagger}=\left(\begin{array}{cc}
H_{A}-E & -H_{A B} \\
H_{A B}^{\dagger} & H_{B}-E
\end{array}\right)
$$

Considering the block matrix $\Delta$ and $\Delta^{\dagger}$, when $\left(H_{B}-E\right)$ is invertible, employing the Leibniz formula, we have

$$
\begin{aligned}
\operatorname{det}(\Delta)= & \operatorname{det}\left(H_{B}-E\right) \operatorname{det}\left[\left(H_{A}-E\right)\right. \\
& \left.-H_{A B}\left(H_{B}-E\right)^{-1}\left(-H_{A B}\right)^{\dagger}\right],
\end{aligned}
$$

and also

$$
\begin{aligned}
\operatorname{det}\left(\Delta^{\dagger}\right)= & \operatorname{det}\left(H_{B}-E\right) \operatorname{det}\left[\left(H_{A}-E\right)\right. \\
& \left.-\left(-H_{A B}\right)\left(H_{B}-E\right)^{-1} H_{A B}^{\dagger}\right] .
\end{aligned}
$$

Then we have

$$
\operatorname{det}(\Delta)=\operatorname{det}\left(\Delta^{\dagger}\right)=\left[\operatorname{det}\left(\Delta^{T}\right)\right]^{*}=[\operatorname{det}(\Delta)]^{*},
$$

i.e., det $(\Delta)$ is real. Such feature arises from the special structure of the matrix $\Delta$ in the form

$$
\left(\begin{array}{cc}
\mathcal{A} & \mathcal{C} \\
-\mathcal{C}^{\dagger} & \mathcal{B}
\end{array}\right)
$$

with $\mathcal{A}$ and $\mathcal{B}$ being Hermitian matrices.

Matrix $M_{i j}\left(i, j \in\left[1, N_{A}\right]\right)$ is obtained from $\Delta$ by eliminating its $i$ th row and $j$ th column, which has the form

$$
\left(\begin{array}{ll}
\mathcal{A}^{\prime} & \mathcal{C}^{\prime} \\
\mathcal{D}^{\prime} & \mathcal{B}
\end{array}\right)
$$

and accordingly $\left(M_{j i}\right)^{\dagger}$ has the form

$$
\left(\begin{array}{cc}
\mathcal{A}^{\prime} & -\mathcal{C}^{\prime} \\
-\mathcal{D}^{\prime} & \mathcal{B}
\end{array}\right)
$$


Here, $\mathcal{A}^{\prime}$ is the matrix by eliminating the $i$ th row and $j$ th column from $\mathcal{A}$, while $\mathcal{C}^{\prime}\left(\mathcal{D}^{\prime}\right)$ is the matrix by eliminating the $i$ th row $(j$ th column) from $\mathcal{C}(\mathcal{D})$. By similar procedure in obtaining Eqs. (53) and (54), we have

$$
\operatorname{det}\left(M_{i j}\right)=\operatorname{det}\left[\left(M_{j i}\right)^{\dagger}\right]
$$

and then

$$
\operatorname{det}\left(M_{i j}\right)=\left[\operatorname{det}\left(M_{j i}\right)\right]^{*} \text {. }
$$

Together with Eq. (15), we have $\left(\Delta^{-1}\right)_{i j}=\left(\Delta^{-1}\right)_{j i}^{*}$, and yield Eq. (22).

\section{ACKNOWLEDGMENTS}

We acknowledge the support of the CNSF (Grant No. 10874091) and National Basic Research Program (973 Program) of China under Grant No. 2012CB921900.
[1] C. M. Bender, and S. Boettcher, Phys. Rev. Lett. 80, 5243 (1998).

[2] C. M. Bender, S. Boettcher, and P. N. Meisinger, J. Math. Phys. 40, 2201 (1999).

[3] P. Dorey, C. Dunning, and R. Tateo, J. Phys. A: Math. Gen. 34, L391 (2001); P. Dorey, C. Dunning, and R. Tateo, J. Phys. A: Math. Gen. 34, 5679 (2001).

[4] C. M. Bender, D. C. Brody, and H. F. Jones, Phys. Rev. Lett. 89, 270401 (2002).

[5] A. Mostafazadeh, J. Math. Phys. 43, 3944 (2002).

[6] A. Mostafazadeh and A. Batal, J. Phys. A: Math. Gen. 37, 11645 (2004).

[7] A. Mostafazadeh, J. Phys. A: Math. Gen. 36, 7081 (2003).

[8] H. F. Jones, J. Phys. A: Math. Gen. 38, 1741 (2005).

[9] A. Mostafazadeh, J. Phys. A: Math. Gen. 38, 6557 (2005).

[10] A. Mostafazadeh, J. Phys. A: Math. Gen. 39, 10171 (2006).

[11] A. Mostafazadeh, J. Phys. A: Math. Gen. 39, 13495 (2006).

[12] Y. N. Joglekar, D. Scott, M. Babbey, and A. Saxena, Phys. Rev. A 82, 030103(R) (2010).

[13] D. D. Scott and Y. N. Joglekar, Phys. Rev. A 83, 050102(R) (2011).

[14] L. Jin and Z. Song, Phys. Rev. A 80, 052107 (2009).

[15] O. Bendix, R. Fleischmann, T. Kottos and B. Shapiro, Phys. Rev. Lett. 103, 030402 (2009).
[16] K. Zhou, Z. Guo, J. Wang and S. Liu Opt. Lett. 35, 2928 (2010).

[17] Y. D. Chong, Li Ge, Hui Cao and A. D. Stone, Phys. Rev. Lett. 105, 053901 (2010).

[18] S. Longhi, Phys. Rev. A 82, 031801(R) (2010); Phys. Rev. Lett. 105, 013903 (2010).

[19] L. Jin and Z. Song, Phys. Rev. A 81, 032109 (2010); Commun. Theor. Phys. 54, 73 (2010).

[20] Z. Ahmed, Phys. Lett. A 324, 152 (2004).

[21] M. Znojil, J. Phys. A: Math. Gen. 39, 13325 (2006); Phys. Rev. D 78, 025026 (2008); Phys. Rev. D 80, 045009 (2009).

[22] H. F. Jones, Phys. Rev. D 76, 125003 (2007); Phys. Rev. D 78, 065032 (2008).

[23] S. V. Dmitriev, S. V. Suchkov, A. A. Sukhorukov, and Y. S. Kivshar Phys. Rev. A 84, 013833 (2011).

[24] L. Jin and Z. Song, Phys. Rev. A 81, 022107 (2010).

[25] A. Guo, G. J. Salamo, D. Duchesne, R.Morandotti, M. Volatier-Ravat, V. Aimez, G. A. Siviloglou, and D. N. Christodoulides, Phys. Rev. Lett. 103, 093902 (2009).

[26] T. Kottos, Nature Phys. 6, 166 (2010).

[27] C. E. Rüter, K. G. Makris, R. El-Ganainy, D. N. Christodoulides, M. Segev, and D. Kip, Nature Phys. 6, 192 (2010). 